\title{
Adoption of Telemedicine -need of the hour
}

\section{Dear Editor,}

I would like to describe our experience in telemedicine for the provision of health care in the Northern Territory of Australia and its relevance for the state of Jammu and Kashmir. Northern Territory is a large landmass spread over 1.4 million square kilometers with a population of only 250,000. Major healthcare facilities are located in cities of Darwin and Alice Springs with smaller hospitals in Katherine, Gove, and Tennant Creek. Many small communities are located in remote areas with limited access to health care. We have adopted telemedicine more than a decade ago and gradually scaled up the services which came handy during the latest pandemic due to SARS-CoV-2.

Telemedicine encompasses the delivery of health care using a variety of telecommunication tools. In addition to improved access to health care, telemedicine can reduce the cost and improve convenience. Specialists triage suitable referrals to teleclinics. The patient/carer is informed of the appointment and consent is obtained. The clinic staff procures the relevant investigation reports to be ready at the time of the consult. To run an efficient and effective teleclinic it is essential to have appropriately trained administrative support. Chronic disease management, wherein physical examination is not crucial for decision making, is well suited for telemedicine. Teleclinics are also suitable to assess the need for patients to come into the hospital as well as provide pre-operative advice and preparation prior to planned elective surgical procedures.

Multidisciplinary case conferences with patients, family members, general practitioners, and hospital-based specialists work well to formulate an individualized management plan for complex patients. People living in

\begin{tabular}{l|l}
\hline & Access this article online \\
\hline Website: & \\
\hline
\end{tabular}

remote locations in Jammu and Kashmir are likely to experience difficulty in reaching Srinagar and Jammu due to limited public transportation options, hilly terrain, heavy snow in winter, political upheaval and the frequent imposition of restriction on movement by the law enforcement authorities.

Patient factors that determine the feasibility of telemedicine include access to a telephone (landline or mobile), internet connection with sufficient bandwidth, hearing and communication ability, and ability to use video applications on a mobile phone (smartphone) or computer for video consultations. Many patients in remote communities have low literacy and limited access to internet services in addition to sub-optimal communication skills. These patients access teleclinics by going to the nearest community health center from where they can link up to the health care providers in major centers. We involve interpreter services, family members, health care workers in the community health center in the discussions for patients who have poor health literacy, hearing difficulties, or whose first language is other than English. Seamless link up with pathology, radiology, and pharmacy services to enable electronic test requests, test/imaging reports and prescriptions will improve the client experience.

Telehealth can play a meaningful role in natural disasters and pandemics by helping in "forward triage" before patients arrive in the emergency department as well as directing patients to the hospitals with bed capacity ${ }^{1,2}$. The provision of telehealth for patients in pandemic lockdown or quarantine ensures delivery of necessary care while protecting health care staff and the community from exposure. We have converted most of the scheduled clinic

(9) (1) This is an open-access article distributed under the terms of the Creative Commons Attribution License (CC BY 4.0), which permits unrestricted use, distribution, and reproduction in any medium, provided the original author and source are credited. 
Chitturi S; Adoption of Telemedicine -need of the hour

visits to teleclinic visits allowing for both patients and clinicians to stay at home and provide uninterrupted clinical care. This is important to reduce the emergency department presentations by patients with non-COVID related illnesses.

Innovation and rapid scaling up of telehealth services with improved infrastructure and staff and patient training to support the telehealth service can go a long way to provide more equitable and cost-effective health care to the neediest. Medicare coverage for telehealth services was rapidly expanded in Australia which is likely to have farreaching effects on reshaping the healthcare landscape. For certain health conditions, telehealth can be the preferred first option with in-person consultations being the second or third option to meet the patient's health care needs ${ }^{3}$.

\section{REFERENCES}

1. Lurie N, Carr BG. The role of telehealth in the medical response to disasters. JAMA Intern Med 2018; 178:745-746.

2. Hollander JE, Carr BG. Virtually perfect? Telemedicine for COVID-19. N Engl J Med 2020.

3. Duffy S, Lee TH. In-person health care as option B. N Engl J Med 2018; 378:104-106.

Dr. Sridhar Chitturi MD, DM, FRACP

Senior Consultant Endocrinologist Royal Darwin Hospital Tiwi, NT 0810, Australia Email: sridharchitturi@gmail.com 